\title{
CAMBIOS EN LA FERTILIDAD DEL SUELO, PRODUCCIÓN DE BIOMASA Y BALANCE DE NITRÓGENO EN SISTEMAS AGROFORESTALES CON CAFÉ EN NICARAGUA
}

\author{
Rodolfo Munguía-Hernández ${ }^{1}$, J eremy Haggar², Alejandro Silvio-Ponce ${ }^{3}$ \\ ${ }^{1}$ Universidad Nacional Agraria (UNA), Managua, Nicaragua / Rodolfo.Munguia@una.edu.ni \\ ${ }^{2}$ Centro Agronómico Tropical de Investigación y Educación (CATIE), Managua, Nicaragua. \\ ${ }^{3}$ Instituto Nicaragüense de Tecnología Agropecuaria (INTA) / Municipio de Masatepe, Masaya, Nicaragua.
}

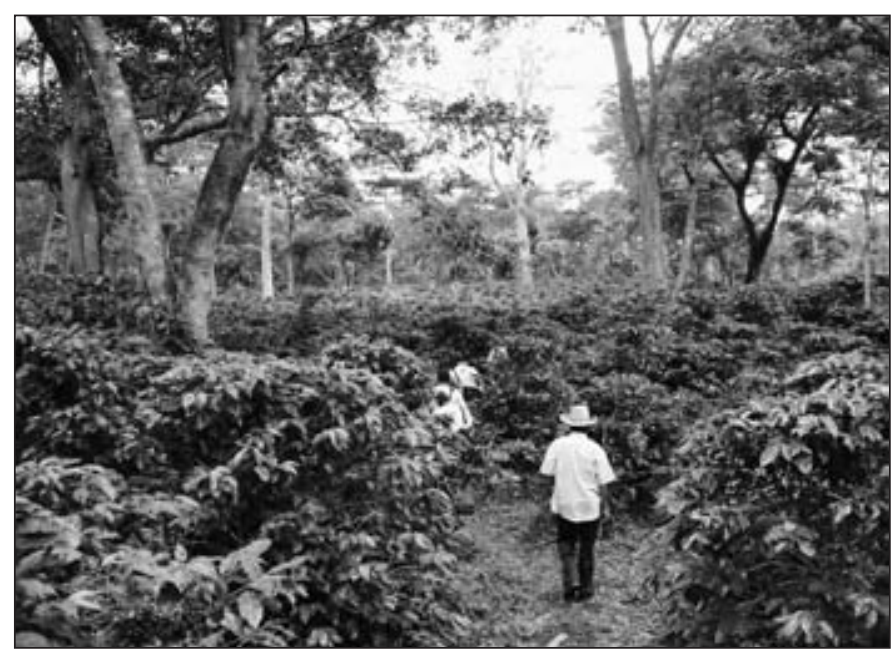

\section{RESUMEN}

Se estableció un experimento de un sistema agroforestal con el cultivo de café en el año 2001 en el Municipio de Masatepe, Nicaragua; estableciéndose en las parcelas principales especies arbóreas leguninosas y no leguminosas y en las subparcelas niveles de fertilizantes orgánicos y químicos aplicados a diferentes cantidades para el manejo de la fertilidad del suelo, enfermedades, plagas insectiles y malezas, con el propósito de establecer los cambios en la fertilidad del suelo, la producción de residuos vegetales y el balance de Nitrógeno. Para la determinación de las variables del suelo, mineralización del nitrógeno, carbono y biomasa microbiana se extrajo por parcela experimental un volumen de suelo para constituir una muestra compuesta. Para la producción de biomasa fueron muestreados dos árboles por parcela y determinados los contenidos de $\mathrm{N}$; fue considerada la cantidad de $\mathrm{N}$ aportados por la adición de fertilizantes orgánicas y químicos. El $\mathrm{N}$ extraído por medio de la cosecha (grano uva) fue determinado para incluirlo en el balance. Los resultados muestran que se produce una menor acidez del suelo, y estadísticamente diferente en el Orgánico Intensivo comparado al manejo Convencional Moderado, y similar comportamiento en Fósforo, Potasio y la biomasa microbiana. En tanto la CIC, el N y el C total, resultó menor en el 2004 con respecto al 2001, en ambos años no hubo diferencias estadísticas entre los tratamientos. La tasa de mineralización del $\mathrm{N}$ es menor en el tratamiento a pleno sol con fertilizante

\begin{abstract}
A coffee agroforestry experiment was established in 2001 in the Municipality of Masatepe, Nicaragua; main treatments were legume and non-legume arboreal species with subplots of intensive and moderate use of organic and conventional management of fertility, pests, diseases and weeds. Soil chemical characteristics, mineralization of nitrogen, carbon and microbial biomass were measured in each plot for a composite soil sample. To measure biomass production two trees per plots were pruned and $\mathrm{N}$ content determined. The $\mathrm{N}$ balance was calculated by determining the extracted $\mathrm{N}$ in the coffee berries, firewood production compared to the amount of $\mathrm{N}$ contributed by organic and chemical fertilizers. The results show that acidity fell under intensive organic management compared to moderate conventional, while phosphorus, potassium and the microbial biomass were all higher under organic than conventional. The CIC, total $\mathrm{N}$ and total C, were all lower in the 2004 with respect to the 2001. In both years there were no statistical differences between the treatments. The rate of mineralization of the $\mathrm{N}$ was lower under the sun treatment total with respect to the treatments with arboreal shade; and higher the intensive organic management than conventional management. The amounts of residues produced by pruning are greatest for Inga laurina, nevertheless, in the year when trees were thinned abebuia. rosea it surpasses it. The nitrogen balance was negative for
\end{abstract}


químico con respecto a los tratamientos con sombra arbórea; mientras que los fertilizantes orgánicos muestran ser diferentes estadísticamente con respecto a fertilizantes químicos aplicados al suelo. Las cantidades de residuos vegetales producidos por la poda es dado por Inga. laurina, sin embargo, en el raleo Tabebuia. rosea la supera en esta actividad. El balance de Nitrógeno resulta negativo en los niveles de fertilizante Moderado Convencional y Orgánico Moderado dado que las extracciones por cosecha de los frutos de café y extracciones de leña son superiores a las adiciones de $\mathrm{N}$ vía fertilización provocando un empobrecimiento de este elemento mineral en el suelo.

Palabras clave: Orgánico, Convencional, Leguminosas, Balance de Nitrógeno, Biomasa, Simarouba glauca, Tabebuia rosea, Inga laurina, Samanea saman
Moderate Conventional and Organic Moderate management under Inga. laurina since the extractions by coffee berries harvest and firewood are superior to the additions of $\mathrm{N}$ via fertilization causing an impoverishment of this mineral element in the ground.

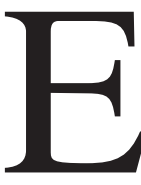
n América Latina en 1990, se ha estimado que más de la mitad de las plantaciones cafetaleras ha cambiado a un manejo intensivo monocultural bajo una especie arbórea como sombra, o a plena exposición solar (Perfecto et al, 1996). En éstos sistemas se centraron esfuerzos en aumentar los rendimientos a través del empleo de tecnologías, que incluyó el uso de variedades productivas, algunas con resistencia o tolerancia a enfermedades; el uso de plaguicidas sintéticos y fertilizantes químicos para maximizar la producción (Haggar, 2005).

La aplicación de éstos insumos agrícolas obvió la importancia de los procesos ecológicos que favorecen la estabilidad productiva y más aún a la descontaminación permanente en el ambiente (aire, suelo, agua) que ejercen sus funciones ambientales dentro del sistema, lo cual ha sido criticado; además de tener una rentabilidad riesgosa y la poca accesibilidad (Haggar and Staver, 2001).

En los sistemas agroforestales los árboles son considerados como un componente integral para el logro de la sustentabilidad de los recursos existentes en el sistema; mediante las características morfológicas, fisiológicas y fenológicas que permiten modificar las condiciones de microclima (temperatura, humedad relativa, precipitación, viento y radiación solar) (Gliessman, 2002).

En un ecosistema de café, los árboles contribuyen al incremento de la materia orgánica del suelo por medio de los aportes naturales ocurridos por caída de hojarasca, residuos de podas y raíces muertas, que mantienen o aumentan la fertilidad (Palm y Sánchez, 1990; Mofongoya et al, 1998; Basavaraju y Gururaja, 2000) e incrementando a largo plazo la productividad en el tiempo, reduciendo los altos costos de manejo y de insumos (Nair, 1984; López, 1993; Ramírez, 1993; Labrador y Altieri, 2001).

La variabilidad de los materiales orgánicos permite a la materia orgánica ser un producto de amplio espectro, desarrollando influencias como regulador de las fitohormonas en la planta (Labrador, 1996). Es esencial para la fertilidad y disponibilidad natural de nutrimentos al suelo, esto puede enfocarse desde dos puntos de vista: como fuente directa de macro y micro nutrientes en proceso de mineralización, y por la participación de la fracción orgánica (enmiendas orgánicas) en procesos que mejoran la disponibilidad (Meléndez, 2004).

El propósito del presente trabajo fue establecer los cambios en la fertilidad del suelo ocurridos debido a la interacción del los árboles, cafetos y manejo del sistema agroforestal; además de cuantificar los aportes de biomasa según sistemas de árboles como sombra en el cafetal y el balance del nitrógeno a partir de las aportaciones y exportaciones de éste elemento mineral.

\section{MATERIALES Y MÉTODOS}

Se estableció un experimento de café (var Pacas) en 2001 en el Centro Jardín Botánico ( $11^{\circ} 54^{\prime} \mathrm{LN}, 86^{\circ} 09^{\prime}$ ) y Centro Experimental Campos Azules (12 $19^{\prime}$, 86 $04^{\prime}$ ) en el Municipio de Masatepe, Departamento de Masaya, Nicaragua; es una zona baja (455 msnm) y seca (1386 mm de precipitación) con suelos fértiles; $\mathrm{pH}$ de 5.7 a 6.0; Materia orgánica 10 a 12 \% y CIC de 54 a 57 (Haggar 2005).

En parcelas principales se incluyen diferentes combinaciones de árboles para formar un gradiente de fijación de nitrógeno y combinaciones contrastantes de fenología y de forma de dosel (Tabla 1); y en suparcelas se manejan los niveles de insumos para el manejo de la 
fertilidad, de plagas y malezas formado por dos manejos orgánicos y dos convencionales de uso de insumos intensivos y moderados (Tabla 2).

La siembra de los árboles fue 4 x $3.75 \mathrm{~m}$, raleados el $50 \%$ de la densidad inicial a los $5-6$ años de edad, cada año se realizaron regulaciones de sombra, especialmente para I. laurina (Guabilla). Al inicio del experimento se estableció sombra temporal de Ricinus communis (higuerilla) en los tratamientos no-leguminosas y Cajanus cajan (Gandul) con leguminosas. La siembra del café (Coffea arabica L.) fue a 2 x $1.25 \mathrm{~m}$.

Para determinar los cambios ocurridos en la

Tabla 1. Especies arbóreas usadas para la comparación de sistemas de café, Masatepe, Nicaragua.

\begin{tabular}{lllll}
\hline Especie arbórea & Fenología & Forma dosel & Fijador de N & Uso \\
Simarouba glauca (Acetuno) & Perennifolia & Alto estrecho & No & Madera \\
Tabebuia rosea (Roble macuelizo) & Caducifolia & Alto estrecho & No & Madera \\
Samanea saman (Genízaro) & Perennifolia & Alto abierto & Sí & Madera \\
Inga laurina (Guabilla) & Perennifolia & Bajo abierto & Sí & Servicio
\end{tabular}

Tabla 2. Niveles de insumos para el manejo de la fertilidad y plagas en la comparación de sistemas de producción de café, Masatepe, Nicaragua.

\begin{tabular}{|c|c|c|c|c|}
\hline \multirow{2}{*}{ Insumos } & \multicolumn{2}{|c|}{ Insumos orgánicos } & \multicolumn{2}{|l|}{ Insumos químicos } \\
\hline & $\begin{array}{l}\text { Orgánico } \\
\text { moderado } \\
(\mathrm{OM})\end{array}$ & $\begin{array}{l}\text { Orgánico } \\
\text { Intensivo (OI) }\end{array}$ & $\begin{array}{l}\text { Convencional } \\
\text { moderado (CM) }\end{array}$ & $\begin{array}{l}\text { Convencional } \\
\text { intensivos (CI) }\end{array}$ \\
\hline $\begin{array}{l}\text { Enmiendas del } \\
\text { suelo }\end{array}$ & Pulpa de café & $\begin{array}{l}\text { Pulpa de café, } \\
\text { gallinaza y piedra } \\
\text { mineral molida. }\end{array}$ & $\begin{array}{l}\text { Fertilizantes } \\
\text { químicos a la mitad } \\
\text { de nivel CI. }\end{array}$ & $\begin{array}{l}\text { Fertilizantes } \\
\text { químicos }\end{array}$ \\
\hline Enfermedades & No & $\begin{array}{l}\text { Aplicaciones } \\
\text { foliares de } \\
\text { botánicos y } \\
\text { biológicos. }\end{array}$ & $\begin{array}{l}\text { Uso infrecuente } \\
\text { de fungicidas } \\
\text { comerciales. }\end{array}$ & $\begin{array}{l}\text { Uso calendarizado } \\
\text { de fungicidas } \\
\text { comerciales. }\end{array}$ \\
\hline $\begin{array}{l}\text { Plagas } \\
\text { insectiles }\end{array}$ & $\begin{array}{l}\text { Pepena en } \\
\text { postcosecha }\end{array}$ & $\begin{array}{l}\text { Aplicaciones } \\
\text { foliares de } \\
\text { biológicos } \\
\text { y prácticas } \\
\text { manuales. }\end{array}$ & $\begin{array}{l}\text { Prácticas manuales } \\
\text { y uso infrecuente } \\
\text { de insecticidas } \\
\text { comerciales. }\end{array}$ & $\begin{array}{l}\text { Prácticas manuales } \\
\text { y uso de insecticidas } \\
\text { comerciales }\end{array}$ \\
\hline Malas hierbas & $\begin{array}{l}2 \text { a } 4 \\
\text { desyerbas } \\
\text { manuales }\end{array}$ & $\begin{array}{l}\text { Manejo selectivo } \\
\text { con prácticas } \\
\text { manuales en } \\
\text { la calle y carril } \\
\text { limpio. }\end{array}$ & $\begin{array}{l}\text { Manejo selectivo } \\
\text { con prácticas } \\
\text { manuales y } \\
\text { herbicidas en la calle } \\
\text { y carril limpio. }\end{array}$ & $\begin{array}{l}\text { Suelo desnudo con } \\
\text { herbicidas }\end{array}$ \\
\hline
\end{tabular}

fertilidad del suelo se extrajo una muestra de suelo de 0 a $10 \mathrm{~cm}$ en seis puntos distribuidos al azar por parcela experimental (subparcela), se homogenizaron y una muestra compuesta fue entregada al laboratorio de suelos de la Universidad Nacional Agraria (UNA) para determinar los contenidos de materia orgánica, acides del suelo, contenidos de N, P, K, Ca y Mg, según Anderson and Ingram, (1993); el presente muestreo fue realizado en siete de los tratamientos.

Por medio del uso del método anaeróbico (Anderson and Ingram 1993), y con el mismo procedimiento de muestreo de suelo explicado anteriormente en todos los tratamientos, se determinó las tasas de mineralización de $\mathrm{NH}_{4}^{+}$a los 0 y 7 días; cada muestra fue puesta en recipiente hermético. Adicionalmente a las muestras de suelo se les determinó la biomasa microbiana.

A los árboles de Tabebuia rosea (roble) y a Simarouba glauca (Acetuno) se les efectuó poda de elevación y anualmente a Inga. laurina (Guabilla) poda de descentralización. En cada momento de realización de esta actividad se procedió al pesado del material verde por componente (hojas verdes, ramas finas menores de $2 \mathrm{~cm}$ de diámetro, ramas mayores de 2 $\mathrm{cm}$ y troncos) en dos árboles por especie y tratamiento, de ello se obtuvo la materia seca. En 2005 fueron raleados todas las especies excepto Samanea saman (genizaro) establecida dos años posteriores y sustituyendo a Enterolobium c i c loc a r p u m (Guanacaste). De igual manera dos árboles fueron pesados por componentes y se determinó peso seco y posteriormente el contenido de N. Con respecto al balance de nitrógeno se tomaron los registros anuales de las aplicaciones de $\mathrm{N}$ en forma de fertilizantes químicos y orgánicos según el manejo en las parcelas, de las cosechas anuales y la biomasa seca producto de la extracción por leña se cuantificó el Nitrógeno.

Se realizó análisis de varianza y separación de medias (LSD) a las variables en estudio del suelo y 
para el balance de nutrientes el análisis fue descriptivo a través de los promedios.

\section{RESULTADOS Y DISCUSIÓN}

Cambios en las propiedades químicas del suelo. Los resultados indican que el pH del suelo en el año 2004, fueron superiores estadísticamente $(\mathrm{P}>0.0003)$ en los tratamientos de sombra con manejo Orgánico Intensivo (OI) cerca de la neutralidad (Tabla 3) comparado al manejo Convencional Moderado (CM); mientras que en el 2001 no muestra diferencias estadísticas.

Con respecto a la Capacidad de Intercambio Cationico (CIC), se muestran la tendencia mostrada en el 2004, a una reducción de cationes debido muy probablemente a la absorción de nutrientes principalmente bases del suelo por el cafetal y los árboles de sombra (Tabla3).

Tabla 3. Acidez (pH) y Capacidad de Intercambio Catiónico (CIC) bajo manejo Orgánico y Convencional de 0 a 10 cm de profundidad de suelo

\begin{tabular}{lllll}
\hline $\begin{array}{l}\text { Trata- } \\
\text { miento }\end{array}$ & $\mathrm{pH}\left(\mathrm{H}_{2} \mathrm{O}\right)$ & \multicolumn{3}{c}{$\begin{array}{l}\text { CIC (meq / 100 g de } \\
\text { suelo) }\end{array}$} \\
\hline & 2001 & 2004 & 2001 & 2004 \\
IlSg OI & 5.8 & $6.4 \mathrm{a}$ & 70.2 & 41.5 \\
IlSs OI & 5.9 & $6.5 \mathrm{a}$ & 71.4 & 39.2 \\
SgTr OI & 5.9 & $6.4 \mathrm{a}$ & 70.0 & 39.0 \\
IlSg CM & 5.7 & $5.6 \mathrm{~b}$ & 69.4 & 40.6 \\
IlSs CM & 5.9 & $5.9 \mathrm{~b}$ & 69.8 & 38.0 \\
SgTr CM & 5.8 & $5.9 \mathrm{~b}$ & 68.3 & 34.9 \\
Psol CM & 5.6 & $5.8 \mathrm{~b}$ & 67.4 & 35.5 \\
Pr>F & 0.6785 & 0.0003 & 0.8187 & 0.1826 \\
\hline
\end{tabular}

De acuerdo al análisis químico, el nitrógeno y el potasio en el suelo muestran una reducción de su disponibilidad en el 2004, no así para el caso del fósforo que incrementó su concentración. Tanto el fósforo y potasio fueron superior estadísticamente a favor del manejo orgánico en las parcelas que el manejo convencional. La mayor concentración de fósforo mostrada en las parcelas a plena exposición solar se debe a la ausencia de árboles de sombra por lo que su disponibilidad se incrementa en el tiempo (Tabla 4).

Los contenidos presentados en el análisis de Carbono del suelo indican una reducción en el 2004, lo cual puede deberse a procesos de descomposición acelerados en este período por la acción de factores como la actividad Sol microbiológica, la cantidad de precipitaciones ocurridas y finalmente por el nulo aporte de materia orgánica (residuos vegetales) provenientes de los árboles de sombra que se encontraban en una fase de crecimiento (Tabla 5).

La masa microbiana determinada muestra que es mayor en los tratamientos manejados con insumos orgánicos y estadísticamente diferentes $(\mathrm{P}>0.04)$ a los tratamientos manejados con fertilizantes químicos (Tabla 5). La disponibilidad de una fuente energética (Carbono) en el suelo por las aportaciones de abonos orgánicos ( 9 ton ha ${ }^{-1}$ año ${ }^{-1}$ de pulpa de café y 7 ton ha ${ }^{-1}$ año ${ }^{-1}$ de gallinaza) y adicionalmente aportes debidos a podas y raleo de árboles de sobra favorecen una mayor actividad biológica incrementando sus poblaciones que fijan en sus organismos diferentes elementos minerales.

Tabla 5. Contenidos de Carbono del suelo y microbiano bajo manejo orgánico y convencional a profundidad de 0 a $10 \mathrm{~cm}$.

\begin{tabular}{llll}
\hline \multirow{2}{*}{ Tratamiento } & \multicolumn{2}{l}{ C total (\%) } & \% C microbiano \\
& 2001 & 2004 & 2004 \\
\hline Il Sg OI & 7.23 & 5.5 & $2.17 \mathrm{a}$ \\
Il Ss OI & 7.23 & 5.8 & $1.68 \mathrm{ab}$ \\
Sg Tr OI & 7.14 & 5.5 & $0.77 \mathrm{c}$ \\
Il Sg CM & 7.15 & 5.6 & $1.48 \mathrm{abc}$ \\
Il Ss CM & 6.51 & 5.0 & $1.17 \mathrm{bc}$ \\
Sg Tr CM & 7.41 & 5.9 & $0.97 \mathrm{bc}$ \\
Psol CM & 6.34 & 4.8 & $1.29 \mathrm{bc}$ \\
\hline Pr $>$ F & & 0.6546 & 0.04 \\
\hline
\end{tabular}

Nitrógeno mineralizado. El nitrógeno, el macro elemento mas importante e indispensable para el crecimiento del cafeto, principalmente en la producción de follaje que favorece la actividad fotosintética,

Tabla 4. Concentraciones de N, P y K del suelo bajo manejo orgánico y

Il : Inga laurina; Ss: Samanea saman; Sg: Simarouba glauca; Tr: Tabebuia rosea; CM: Convencional Moderado; OI: Orgánico Intensivo; Psol: Pleno 
influyen en la producción de ramas laterales productivas, posibilitan una mayor fructificación y mejora rendimiento (Fischersworring y Robkamp, 2001).

El nitrógeno es asimilado por las plantas como ion Amonio $\left(\mathrm{NH}_{4}^{+}\right)$y Nitrato $\left(\mathrm{NO}_{3}^{-}\right)$, de tal manera que estas formas presentes en el suelo en las cantidades necesarias son muy importantes para los cultivos en general.

El proceso de mineralización del nitrógeno está siendo favorecido por la presencia de los árboles de sombra en el sistema de café al compararse con el tratamiento de café a plena exposición solar (Tabla 6), donde las actividades de manejo de plagas del suelo y malezas se realiza aplicando sustancias químicas (insecticidas, fungicidas y herbicidas) los que pueden estar provocando una acción negativa a la mineralización. Este comportamiento mostrado con manejo orgánico se debe a los aportes orgánicos y a los depósitos de material vegetal que es producido por

Tabla 6. Tasa de mineralización por tipos de sombra

\begin{tabular}{lll}
\hline Tipo de sombra & \multicolumn{2}{l}{$\begin{array}{l}\text { Tasa de mineralización N } \\
\left(\mathrm{mg} \mathrm{NH}_{4}{ }^{+} \mathrm{kg}^{-1} \text { de suelo) }\right.\end{array}$} \\
\hline Ss Il (Genízaro - Guabillo) & 2002 & 2006 \\
Ss Tr (Genízaro - Roble) & 11,76 & 21,83 \\
Il Sg (Guabillo - Acetuno) & 11,34 & 21,96 \\
Psol (Café sin sombra) & 9,92 & 16,28 \\
Sg Tr (Acetuno - Roble) & 11,31 & 21,19 \\
\hline pr > F & 0,294 & 0,5735 \\
\hline
\end{tabular}

$\mathrm{SS}=$ S. saman; $\mathrm{IL}=$ I. laurina $; \mathrm{TR}=$ T. rosea $;$ Psol $=$ Pleno sol $\mathrm{SG}=$ S. glauca

la actividad de poda y raleo de los árboles y en el caso particular de la especie I. laurina y $S$. saman las que fijan nitrógeno en el suelo; así también, Altamirano (2005) determinó que en las combinaciones donde estaba presente la especie I. laurina la cantidad de residuos fue muy superior al resto de tratamientos.

La misma tendencia a incrementarse con el tiempo es mostrado en el 2006 para el factor niveles de insumos aplicados en cada uno de los tratamientos, siendo superior estadísticamente $(\mathrm{P}>0.0017)$ en los niveles con manejo orgánico en forma de pulpa de café más gallinaza (OI) que contribuyen a una mayor tasa de mineralización del Nitrógeno, siendo mayor cuando se usa mayor cantidad de insumos, lo que permitirá a la planta de café y a los mismos árboles de sombra disponer de una mayor cantidad de este elemento mineral para su nutrición (Tabla 7). La menor tasa de mineralización se obtuvo en el tratamiento donde se aplican una mayor cantidad de
Tabla 7. Tasa de mineralización de nitrógeno en el suelo bajo efecto del nivel de insumo

\begin{tabular}{lll}
\hline & \multicolumn{2}{l}{ Tasa de mineralización } \\
Niveles de insumos & \multicolumn{2}{l}{$\begin{array}{l}\text { suelo) } \\
\text { s. }{ }_{4}^{+} / \mathrm{kg} \text { de }\end{array}$} \\
\hline Convencional Intensivo (CI) & 11,54 & $16,44 \mathrm{c}$ \\
Orgánico Moderado (OM) & 9,09 & $22,28 \mathrm{ab}$ \\
Convencional Moderado (CM) & 11,35 & $19,01 \mathrm{bc}$ \\
Orgánico Intensivo (OI) & 11,71 & $27,06 \mathrm{a}$ \\
\hline pr $>\mathrm{f}$ & 0,1084 & 0,0017 \\
\hline
\end{tabular}

insumos químicos (CI) lo que pueda estar reduciendo la capacidad microbiológica para el desarrollo de este proceso en el suelo.

Producción de materia seca por especie forestal. La biomasa está considerada como un parámetro importante en la caracterización de los ecosistemas, ya que refleja su capacidad de acumular materia orgánica durante un determinado periodo (Sarmiento 1984). Además, al igual que el aumento en la vegetación, es un componente esencial para la estimación del secuestro de Carbono (Eamus y Burrows, 2000).

El manejo anual realizado a los árboles en las diferentes especies, dan como resultado un manejo diferenciado en cuanto a la intensidad de podas de descentralización que se realizó en I. laurina, de elevación en S. glauca; un raleo en las especies mencionadas y $T$. rosea. Como se observa en el año 2004, se podaron I. laurina y S. glauca, la primera especie produjo la cantidad de $1,888.7 \mathrm{~kg} \mathrm{ha}^{-1}$ residuos que se incorporan al suelo, sin embargo, a esta misma especie se extrajo mayor cantidad de leña $(3,104.4 \mathrm{~kg}$ ha $^{-1}$ ) que salen fuera del sistema (Tabla 8).

En tanto, en 2005 se realizó poda fuerte en I. laurina y raleo (33 \% de población inicial) en $S$. glauca y $T$. rosea, donde la producción por efecto de eliminación de T. rosea se produjeron $6,529.7 \mathrm{~kg} \mathrm{ha}^{-1}$ muy superiores a las otras especies y combinada con $S$. glauca producen un total de 9,418.5 $\mathrm{kg} \mathrm{ha}^{-1}$ de materia seca que quedan en el sistema para su incorporación lenta a la materia orgánica al suelo por descomposición de la misma. Con respecto a la remoción de leña de igual manera que en el año anterior la especie I. laurina fue superior en las combinaciones I. laurina + S. glauca y S. saman $+I$. laurina. Sin embargo, cuando se combinan S. glauca + T. rosea no llegan a superar en cantidad de leña extraída a I. laurina (Tabla 8). 
En 2006, se efectuó poda en S. glauca e I. laurina, ésta última aporta similares cantidades de materia seca que en el año anterior por poda y raleo; no obstante, en I. laurina se extrajeron mayores cantidades de materia seca en forma de leña con 3,910.7 $\mathrm{kg} \mathrm{ha}^{-1}$ en presencia de S. glauca; y 6,069.3 kg cuando está presente con S. saman (Tabla 8), debido a que esta última no fue intervenida por poda dado que fue establecida en el segundo año de crecimiento de las otras especies, lo que provocó un desarrollo menor por efecto de competencia de las otras especies.

Balance de Nitrógeno. El balance en 2004, indican valores positivos en todos los tratamientos, sin embargo, el manejo de insumos moderados convencional (CM) y el moderado orgánico (OM) resultan ser los de menor cuantía comparativamente con el resto de niveles de insumo tanto convencional como orgánico. Este comportamiento obedece en principio a las cantidades menores que se aplican en MC comparado con Convencional Intensivo (CI), mientras que en $\mathrm{OM}$ solamente se considera el retorno de pulpa ( 9 ton ha- $\left.{ }^{-1}\right)$ en cantidades similares a las extraídas en la cosecha, a diferencia en el orgánico Intensivo (OI) que además de pulpa se adiciona gallinaza ( 7 ton ha $\left.{ }^{-1}\right)$ que hace que las cantidades de $\mathrm{N}$ sean muy similares al CI (Tabla 9).
En el año 2005, se realizó poda y eliminación del 33 \% de árboles por especie y se muestra mayores valores de Nitrógeno extraídas en forma de leña y relativamente superiores las extracciones debidas a la cosecha con respecto al año anterior; dando un balance negativo en CM y OM, sin embargo, el CI en la combinación formada por $S$. saman mas I. laurina solo la extracción de leña supera la adición de $\mathrm{N}$ a través de la fertilización nitrogenada. El balance negativo oscila entre 51 a $98 \mathrm{~kg}$ ha-1 $^{-1}$ (Tabla 9), lo cual reduce la capacidad del suelo de disponer de éste elemento para el cultivo de café lo cual provocará en el futuro deficiencias nutricionales que impactarán en el rendimiento productivo.

En el 2006, solamente el sistema formado por $S$. saman e I. laurina en los niveles de insumos CM y OM fueron negativos debidos a la cantidad de material vegetal en forma de leña que fueron producidos por manejo de poda y extraídos del sistema agroforestal; en el resto de los tratamientos el balance es positivo (Tabla 9), lo cual permitirá cierta recuperación de la capacidad del suelo de ofertar una cantidad importante de $\mathrm{N}$ para posibilitar una absorción por las plantas de café y de los mismos arboles de sombra.

Tabla 8. Producción de biomasa seca incorporada en el sistema y extraída en forma de leña debido a poda y raleo de árboles de sombra

\begin{tabular}{|c|c|c|c|c|c|c|c|c|c|c|c|}
\hline \multirow{2}{*}{$\begin{array}{l}\text { Manejo de la } \\
\text { sombra }\end{array}$} & \multirow{2}{*}{$\begin{array}{l}\text { Sistema de } \\
\text { sombra }\end{array}$} & \multicolumn{5}{|c|}{ Hojas y ramas $<2$ cm de diámetro $\left(\mathrm{kg} \mathrm{ha}^{-1}\right)$} & \multicolumn{5}{|c|}{ Ramas gruesas y troncos $\left(\mathrm{kg} \mathrm{ha}^{-1}\right)$} \\
\hline & & SG & IL & TR & SS & Total & SG & IL & $\mathrm{TR}$ & SS & Total \\
\hline \multirow{4}{*}{ Poda 2004} & IL SG & 182.1 & 776.9 & & & 959.0 & 179.1 & $1,246.4$ & & & $1,425.5$ \\
\hline & SG TR & 449.3 & & & & 449.3 & 442.4 & & & & 442,4 \\
\hline & SS IL & & $1,888.7$ & & 118.7 & $2,007.4$ & & $3,104.4$ & & 96.3 & $3,200.7$ \\
\hline & SS TR & & & & 101.4 & 101.4 & & & & 82.3 & 82.3 \\
\hline \multirow{4}{*}{$\begin{array}{l}\text { Raleo y poda } \\
2005\end{array}$} & IL SG & 577.9 & $2,892.9$ & & & $3,470.8$ & $1,537.3$ & $5,058.5$ & & & $6,595.8$ \\
\hline & SG TR & $2,888.8$ & & $6,529.7$ & & $9,418.5$ & $3,378.2$ & & $3,977.0$ & & $7,355.2$ \\
\hline & SS IL & & $3,809.0$ & & & $3,809.0$ & & $7,578.3$ & & & $7,578.3$ \\
\hline & SS TR & & & & & & & & & & \\
\hline \multirow{4}{*}{ Poda 2006} & IL SG & & 3,287.9 & & & $3,287.9$ & & $3,910.7$ & & & $3,910.7$ \\
\hline & SG TR & 263.1 & & & & 263.1 & 230.7 & & & & 230.7 \\
\hline & SS IL & & 3,398.9 & & & 3,398.9 & & 6,069.3 & & & $6,069.3$ \\
\hline & SS TR & - & - & - & - & - & - & - & - & - & - \\
\hline
\end{tabular}

SG: Simaruba glauca (Acetuno); IL: Inga laurina (Guava); SS: Samanea saman (Genizaro); TR: Tabebuia rosea (Roble). 


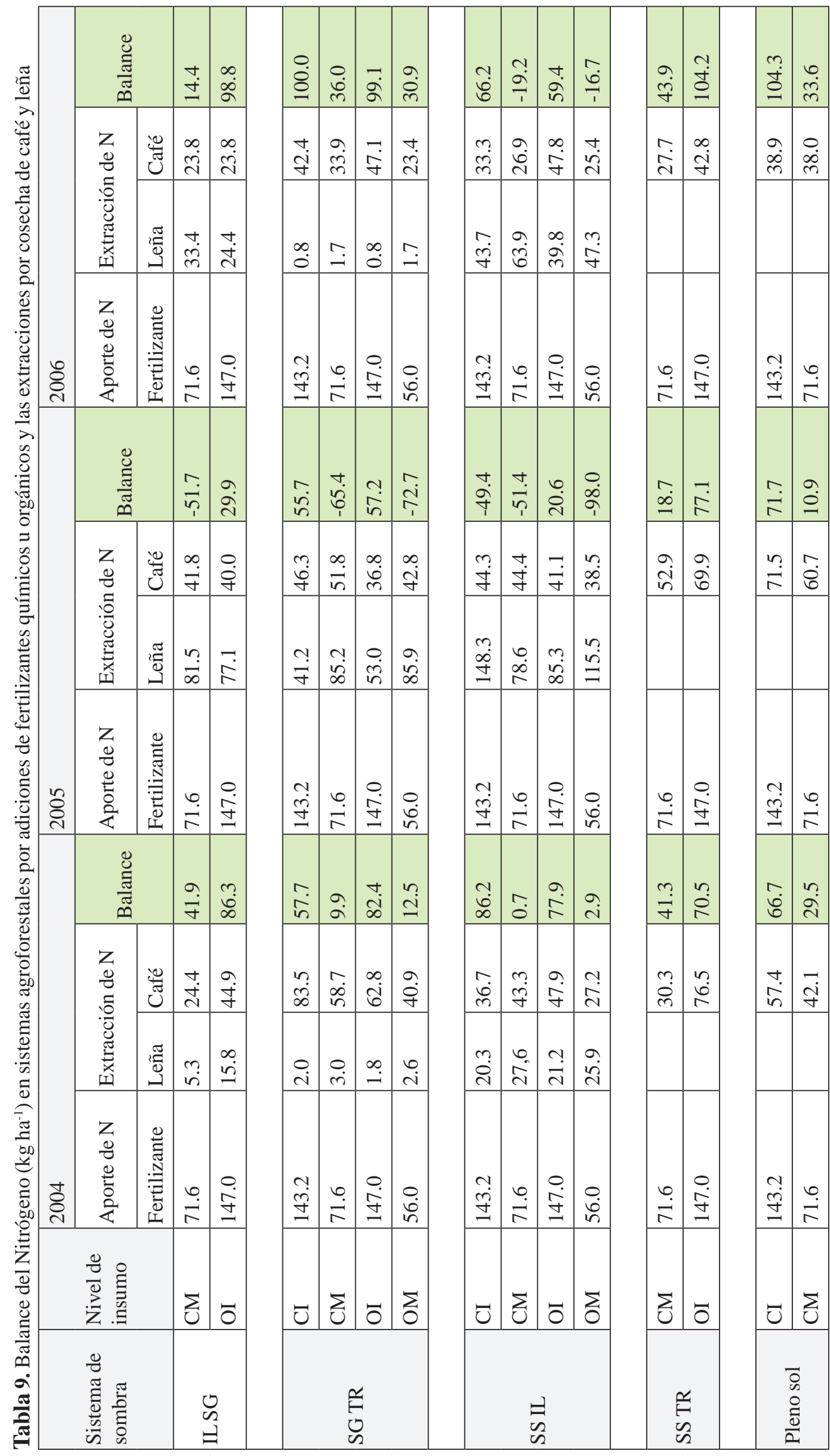

\section{CONCLUSIONES}

La acidez del suelo y la CIC se ven favorecidos por el manejo orgánico de las parcelas.

El nitrógeno y el potasio no muestran diferencias en cuanto al manejo de nutrientes aplicados, sin embargo se reduce su disponibilidad para las plantas; caso contrario el fósforo incrementó su disponibilidad en el 2004 con respecto al 2002 y es mayor aún en las parcelas a plena exposición solar.

El contenido de carbono en el suelo disminuyó en 2004 con respecto al 2002, mientras que la masa microbioana es favorecida por el manejo orgánico de las parcelas.

La mineralización del nitrógeno es mayor en las parcelas donde se realizan deposiciones de residuos vegetales de manera natural y por efecto de poda y raleo; así como en el manejo orgánico donde se aplican sistemáticamente abonos de origen vegetal y animal.

El manejo de poda en I. laurina realizados cada año contribuye de manera sistémica de cantidades importantes de residuos vegetales que se incorporarán a la materia orgánica del suelo; sin embargo, la extracción de leña es fuerte con esta especie arbórea.

El balance de nitrógeno es negativo cuando se extraen cantidades mayores de material vegetal en forma de leña y de cosecha de café, siendo los más afectados los tratamientos Convencional Moderado y 
Orgánico Moderado cuando las adiciones no compensan las extracciones.

\section{AGRADECIMIENTO}

Este trabajo ha sido posible gracias a la colaboración financiera del CATIE a través del proyecto Innovaciones con fondos del NORAD, y de la contribución de los asistentes de apoyo a la investigación Elvin Navarrete y Leddis Navarrete.

\section{REFERENCIAS BIBLIOGRÁFICAS}

Altamirano, J. 2005. Biomasa y nutriente de mantillo en diferentes sistemas de producción de café (Coffea arabica L.) en el Municipio de Masatepe, Departamento de Masaya, Nicaragua. Tesis. Ing. UNA, Managua, Nicaragua $46 \mathrm{p}$.

Anderson., J. M.; Ingram., J. S.I. 1993. Tropical Soil Biology and Fertility. A handbook of Methods. 2da. Edic. CAB International $221 \mathrm{p}$.

Basavaraju, T. B.; Gururaja R.; M. R. 2000. Tree-crop interactions in agroforestry systems; a brief review. Indian Forester, 126(11): $1155-1164$.

Eamus, D.; McGuinness, K.; Burrows, W. 2000. Review of allometric relationships for estimationg woody biomass for Queensland, the northern territory and western Australia. National Carbon Accounting System. 56 p. (Technical report No. 5a).

Gliessman, S. R. 2002. Agro ecología. Procesos ecológicos en agricultura sostenible. CATIE. Turrialba, Costa Rica 359p.

Fischerworring, B; Robkamp, R. 2001. Guía para la caficultura ecológica. Deutsche Gesellschft feir Technische Zusammenarbeit (GTZ) GMBH, Alemania.149 p

Haggar, J. 2005. Investigación regional para una caficultura ecológica y diversificada. CATIE, Turrialba, Costa Rica. 14 p.

Haggar, J.; Staver, Ch. 2001. Sostenibilidad y sinergismo en sistemas agroforestales con café: estudio de interacciones entre plagas, fertilidad del suelo y árboles de sombra. Agroforesteria de las Américas, 8(29): $49-51$.

Labrador, M. J.; Altieri, A. M. 2001. La materia orgánica en los agroecosistemas: Aproximación al conocimiento de la dinámica. la gestión, y la reutilización de la materia orgánica en los agroecosistemas. Ediciones Mundi Prensa. 2da Ed. pp 293.

Labrador, M. J. 1996. La material orgánica en los agrosistemas. Ministerio de Agricultura, Pesca y Alimentación. Editora. Mundi-Prensa. Madrid (España). 174p.

López, Z.1993. Agroforesteria: una alternativa para rescatar ecología. CONCAFE. (3):28-30.

Meléndez, G. 2004. Fracción orgánica del suelo: Residuos orgánicos y materia orgánica del suelo. Centro de Investigaciones Agronómicas. Universidad de Costa Rica. 16p.

Mofongoya, P. L.; Giller, K. E.; Palm, C. A. 1998. Decomposition and nitrogen release patterns of tree prunings and litter. Agroforestry Systems, 38: 77 - 97.

Nair, P. K. R. 1984. Soil Productivity Aspects of Agro forestry: Science and Practice in Agroforestry. International Council for Research in Agro forestry (ICRAF): Nairobi, Kenya. In: Gliessman, S. R. Agroecologia: Procesos ecologicos en agricultura sostenible. / CATIE Turrialba, C. R. p 260-268.

Palm, C. A.; Sánchez, P. A. 1990. Decomposition and nutrient release patterns of the leaves of three legumes. Biotropica 22(4): 330 - 338.

Perfecto, I.; Rice R., A; Greenberg, R. and Var der Voort M. 1996. Shade coffee: A disappearin refuge for biodiversity. Bioscience 46(8): 598 - 608.

Ramírez, L. G. 1993. Producción de Café (Coffea arábica) bajo diferentes niveles de fertilización con y sin sombra.

Sarmiento, G. 1984. Los ecosistemas y la ecosfera. Barcelona, Editorial Blume. 272 p. 